\title{
EVALUATING MBSE POTENTIAL USING PRODUCT AND DEVELOPMENT CHARACTERISTICS - A STATISTICAL INVESTIGATION
}

\author{
M. Schöberl ${ }^{1, \bigotimes}$, E. Rebentisch ${ }^{2}$, J. Trauer ${ }^{1}$, M. Mörtl ${ }^{1}$ and J. Fottner ${ }^{1}$ \\ ${ }^{1}$ Technical University of Munich, Germany, ${ }^{2}$ Massachusetts Institute of Technology, United States of America \\ $\triangle$ max.schoeberl@tum.de
}

\begin{abstract}
As model-based systems engineering (MBSE) is evolving, the need for evaluating MBSE approaches grows. Literature shows that there is an untested assertion in the MBSE community that complexity drives the adoption of MBSE. To assess this assertion and support the evaluation of MBSE, a principal component analysis was carried out on eight product and development characteristics using data collected in an MBSE course, resulting in organizational complexity, product complexity and inertia. To conclude, the method developed in this paper enables organisations to evaluate their MBSE adoption potential.
\end{abstract}

Keywords: model-based systems engineering, complexity, empirical studies, design methodology

\section{Introduction}

As systems grow in scale and complexity, traditional engineering practices deployed in multidisciplinary development programs reach their limits (Sheard et al., 2015). This, along with the advancing digitalization of the workplace, drives the need for organisations to adopt an improved, digital product development methodology (Madni and Sievers, 2018).

Model-based systems engineering (MBSE) is seen as a promising source of such methodologies (Huldt and Stenius, 2019), leveraging the capabilities of models and systems engineering to deal with complex products and development efforts (Friedenthal et al., 2015). Especially organisations dealing with complex products and development programs tend to rely on MBSE in their digital engineering efforts (Madni and Purohit, 2019; Scheeren and Pereira, 2014; Reichwein and Paredis, 2011).

A survey, conducted in an online course on MBSE at the Massachusetts Institute of Technology (MIT) and further elaborated in the course of this paper showed that a majority of engineers think that MBSE should be implemented in their organisations, while stating that their organisations do not use MBSE approaches today (52\% of 347). Combined with only $15 \%$ of 776 participants stating that their organisation's primary mode of capturing system data is predominantly or purely model-based, it indicates a growing number of organisations facing the adoption of MBSE in the near future. INCOSE (2014) formulated its vision for MBSE as being a "standard practice and [...] integrated with other modeling and simulation as well as digital enterprise functions" in 2025.

In order to prepare the management decision whether MBSE should be adopted in the respective organisation, the individual potential of the MBSE adoption has to be initially estimated. Thus, the goal of this paper is to present a methodology on how organisations can initially evaluate their 
preconditions and potential achievable through an adoption of MBSE. The next section describes the current state of research regarding drivers for the adoption of MBSE and current works on the initial potential evaluation.

\section{Related state of research}

According to Madni and Purohit (2019), "several aerospace, automotive, and defense organizations have already begun or are contemplating the transition to model-based systems engineering (MBSE)" to face an "ever-increasing complexity of systems and system development programs". The same reason for the adoption of MBSE can be found in Scheeren and Pereira (2014), who state that efforts to develop MBSE tools and methods have been driven by increasing complexity, making the design of Industrial Automation Systems more challenging.

Reichwein and Paredis (2011) recalls on a more general stage that "systems engineering emerged as a discipline in order to address problems related to the design of complex systems". Friedenthal et al. (2015) demands rigorous and formalized practices to address complexity and highlights that "the practice of systems engineering is undergoing a fundamental transition from a document-based approach to a modelbased approach". Making way for MBSE as "the formalized application of modeling to support systems, requirements, design, analysis, verification and validation activities beginning in the conceptual design phase and continuing throughout development and later life cycle phases" (INCOSE, 2007).

These exemplary quotes underline that there is a unified understanding in the model-based systems engineering community, that complexity drives the adoption of MBSE. It can be assumed that the improvement potential compared to current practices, which can be achieved through implementing MBSE in an organisation, is proportional to the prevailing complexity in that respective environment. Nonetheless, evidence that this assumption is true is not provided in the literature.

Generally, there is little literature on the initial assessment of the preconditions for a high potential MBSE adoption. Madni and Purohit (2019) conducted an economic analysis of model-based systems engineering, sketching the initial assessment. They identified the industries that should derive the most value from the adoption of MBSE. They did so by "parameterizing the MBSE implementation problem in terms of system complexity, regulatory and operational environment complexity, and system lifespan" (Madni and Purohit, 2019).

The approach does not specify why these three factors should be considered for categorization and why these resemble the potential or value accessible. Also, the organisations within the industries are heterogenous and the target audience for potential estimation heuristics are managers or chief systems engineers of these respective organisations. Therefore, an estimation of the achievable potential should be possible on an organisational level, requiring a breakdown of categorization characteristics on this level.

Summing up the related state of research, there is a unified assertion that complexity drives the adoption of MBSE but only one work covers the initial evaluation of achievable potential through an MBSE adoption. Therefore, it is reasonable to clarify the assertion regarding the role of complexity, which and why certain factors should be used for the initial evaluation, as well as to outline an approach to evaluate the achievable MBSE potential on an organisational level.

\section{Research methodology}

To assess the assertion regarding complexity, which and why certain factors should be used for the initial evaluation, as well as to outline an approach to evaluate the achievable MBSE potential on an organisational level, a survey was created and carried out in the MBSE part of the Architecture and Systems Engineering online course program offered at the MIT.

\subsection{Survey}

The survey was conducted in an online course spanning four weeks. Before the course, the participants were asked to provide general information or demographics, such as their company's name, their highest degree obtained, and the years of work experience they have. At the start of the course, their managerial responsibility and their field of expertise were also requested. This information was used to analyse the survey sample. 
In order to analyse which and why certain factors should be used to evaluate the achievable potential through an MBSE adoption, the participants were asked to provide certain characteristics of their organisation's product and development task. The eight characteristics were elaborated in an expert discussion about complexity drivers at MIT and are:

1. Magnitude of your organisation's typical unit cost

2. Part count of your organisation's typical product

3. Source lines of software code in your organisation's typical product

4. Number of different disciplines (functional specialized groups using specific knowledge \& tools distinguishing them from other groups) involved in a typical product development project in your organisation

5. Length of your organisation's typical development cycle

6. Number of full and part time employees involved in a typical product development project of your organisation

7. Number of major changes (budget cut, major change in requirements, new technology added, ...) occurring in a typical product development project of your organisation

8. Number of direct contractor/supplier organisations (in a contractual relationship with your organisation) for your organisation's typical product development project

On top of these characteristics, the primary mode of capturing system data was determined, the use of an MBSE approach at the participant's organisations and the participant's opinion on features that make the aerospace industry a leader in MBSE were in question. Finally, the participants were asked if MBSE should be implemented in their organisation.

\subsection{Sample}

The number of respondents, answering at least one question is 948 . As the participants were free to leave out questions, there is a specific respondent count $(\mathrm{N})$ given for each question. This section outlines the composition of the population of the survey. The composition of the population is discussed to enable the reader to form an opinion on the validity of responses and whose views they reflect.

First, regarding the validity of responses the years of experience and highest degree obtained by the participants of the survey is shown in Figure 1 and analysed below.

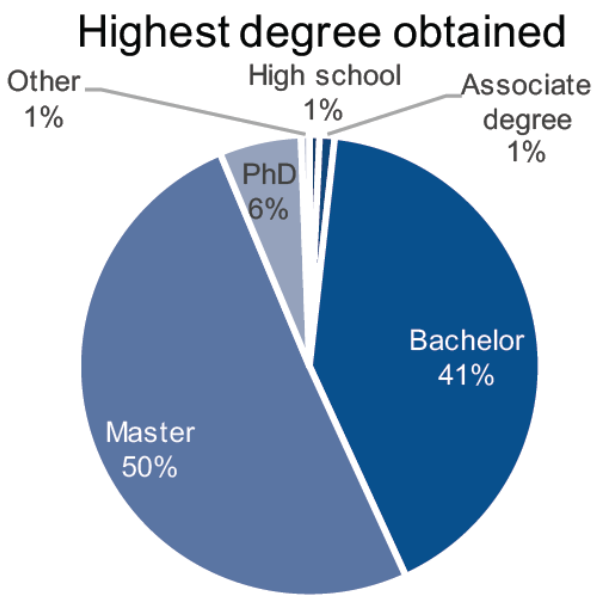

Years of work experience

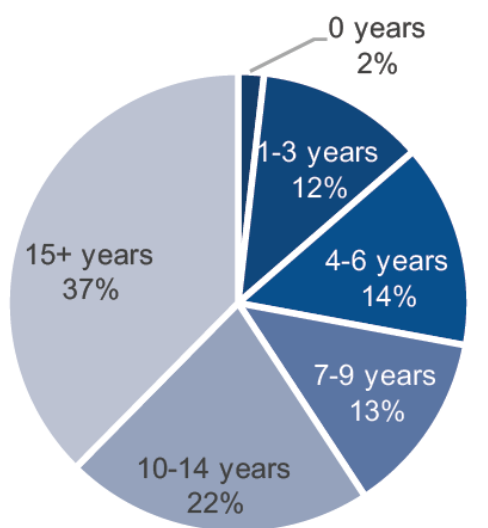

Figure 1. Highest degree obtained $(N=855)$ and years of work experience $(N=888)$ by the survey participants

Most of the survey participants $(97 \%)$ have a bachelor's degree or higher. This indicates a highly educated sample. More than half of the participants have 10 or more years of working experience, which underlines their ability to anticipate future changes in their field of expertise. The data shows that the sample credibility is strongly met.

To understand whose views the survey reflects, the industry, field of expertise, and managerial responsibility of the participants are depicted in Figure 2. 


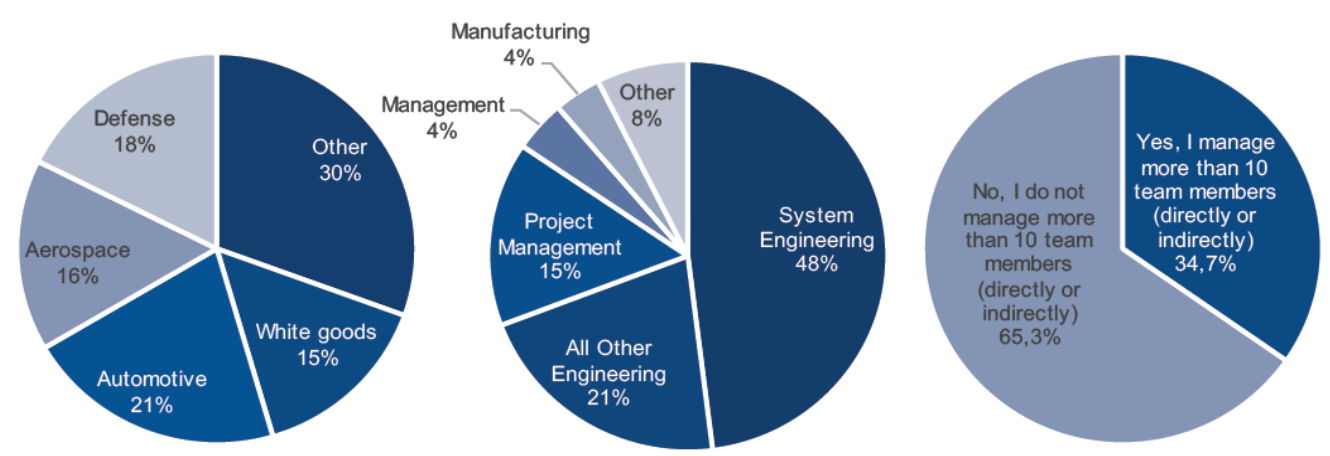

Figure 2. Industry $(N=550)$, field of expertise $(N=754)$ and managerial responsibility $(N=$ 755) of survey participants

The industry has been derived from the company names the participants provided. Almost half of the participants did not provide this information. The four industries with the biggest shares in the population are automotive, defence, aerospace, and white goods. Other industries, like software/IT or consulting were represented but provided a smaller percentage of participants, as the survey was placed in an engineering course.

In another question, the participants were asked which of the above most closely represents their current work. Systems engineering is the predominant field of expertise. Other engineers and project managers represent the biggest shares of all participants not working as system engineers. Management and manufacturing should be highlighted as represented in small shares.

As final indicator for the composition of the survey, the participants have been asked whether they manage more than ten team members (directly or indirectly). $35 \%$ of participants manage more than ten team members directly or indirectly. The majority of participants rather manages smaller teams or has no management responsibilities.

\section{Results}

The data obtained in the survey is analysed in this section with regard to contributions to the justification of the complexity assertion, which and why certain factors should be used for the initial evaluation as well as the creation of an approach to initially evaluate the achievable MBSE potential on an organisational level. All analyses were conducted in the software tool IBM SPSS Statistics.

\subsection{Principal component analysis}

To test the complexity assertion, which and why certain factors should be used for the initial evaluation and to create an approach to initially evaluate the achievable MBSE potential, a principal component analysis (PCA) was conducted on the eight characteristics mentioned in section 3.1 (148 participants in the sample provided answers to all eight questions and form the basis for this analysis). Figure 3 shows the results of the principal component analysis ${ }^{1}$.

Three components (1-3) have obtained eigenvalues over Jolliffe's criterion of 0,7. The matrices in Figure 3 show the unsurpressed (structure matrix) and surpressed (pattern matrix) eigenvectors, also called component loadings, of the correlation matrix (not shown) and the component scores (component score coefficient matrix) for each eigenvalue component respectively. Because an oblique instead of orthogonal rotation is used, the correlations between the components (component correlation matrix) might suppress component loadings in the pattern matrix (e.g. for the characteristics "number of disciplines" and "part count") complicating the interpretation of the results, therefore both are reported.

\footnotetext{
${ }^{1}$ The PCA was conducted on the eight characteristics with oblique rotation (direct oblimin). The Kaiser-MeyerOlkin measure verified the sampling adequacy for the analysis, KMO $=0,868$ ('great' according to Hutcheson and Sofroniou (1999) in Field (2009)), and all KMO values for individual items were > 0,76, which is above the acceptable limit of 0,5 (Field, 2009). Bartlett's test of sphericity $\chi^{2}(28)=453,22, p<$ 0,001 , indicated that correlations between characteristics are large enough for applying PCA. Three components have obtained eigenvalues over Jolliffe's criterion of 0,7 and explained an aggregated $72.97 \%$ of the variance.
} 


\begin{tabular}{|c|c|c|c|c|c|c|c|c|c|}
\hline & \multirow{2}{*}{\multicolumn{3}{|c|}{$\begin{array}{c}\text { Structure Matrix } \\
\text { Component }\end{array}$}} & \multirow{2}{*}{\multicolumn{3}{|c|}{$\begin{array}{c}\text { Pattern Matrix } \\
\text { Component }\end{array}$}} & \multirow{2}{*}{\multicolumn{3}{|c|}{$\begin{array}{c}\text { Component Score } \\
\text { Coefficient Matrix } \\
\text { Component }\end{array}$}} \\
\hline & & & & & & & & & \\
\hline & Org. C. & Prod. C. & Inertia & Org. C. & Prod. C. & Inertia & Org. C. & Prod. C. & Inertia \\
\hline Number of contractors/suppliers & 0,855 & 0,179 & 0,071 & 0,909 & $-0,109$ & $-0,087$ & 0,311 & $-0,122$ & $-0,153$ \\
\hline Number of employees & 0,839 & 0,366 & 0,143 & 0,843 & $-0,137$ & 0,107 & 0,265 & 0,035 & $-0,114$ \\
\hline Number of changes & 0,818 & 0,171 & 0,246 & 0,813 & 0,101 & $-0,041$ & 0,276 & $-0,155$ & 0,056 \\
\hline Number of disciplines & 0,801 & 0,511 & 0,361 & 0,685 & 0,242 & 0,172 & 0,199 & 0,131 & 0,105 \\
\hline Part count & 0,688 & 0,458 & 0,378 & 0,571 & 0,218 & 0,216 & 0,159 & 0,115 & 0,162 \\
\hline Unit cost & 0,179 & 0,841 & 0,424 & $-0,157$ & 0,835 & 0,274 & $-0,117$ & 0,610 & 0,229 \\
\hline Source lines of code & 0,486 & 0,761 & $-0,078$ & 0,300 & 0,724 & $-0,295$ & 0,083 & 0,549 & $-0,388$ \\
\hline \multirow[t]{2}{*}{ Cycle time } & 0,382 & 0,290 & 0,904 & 0,199 & 0,037 & 0,857 & $-0,004$ & $-0,052$ & 0,870 \\
\hline & \multicolumn{3}{|c|}{$\begin{array}{c}\text { Component } \\
\text { Correlation Matrix }\end{array}$} & & & & \multicolumn{3}{|c|}{$\begin{array}{c}\text { Eigenvalues and } \\
\text { Explained Variance }\end{array}$} \\
\hline Component & \multicolumn{3}{|c|}{ Org. C. Prod. C. Inertia } & \multicolumn{3}{|c|}{ Component } & \multicolumn{2}{|c|}{ Org. C. Prod. C. } & Inertia \\
\hline Organisational Complexity & 1,000 & 0,338 & 0,199 & Eigenva & alue & & 3,961 & 1,095 & 0,782 \\
\hline Product Complexity & 0,338 & 1,000 & 0,217 & $\%$ of $\mathrm{Va}$ & riance & & 49,512 & 13,686 & 9,772 \\
\hline Inertia & 0,199 & 0,217 & 1,000 & Cumula & tive $\%$ & & 49,512 & 63,198 & 72,970 \\
\hline
\end{tabular}

Figure 3. Summary of the principal component analysis for the MBSE survey $(\mathbf{N}=148$, in bold: correlation and loading values $>0,4$ as well as component score values $>0,1$, in grey: positive component score values $>0,1$ )

The component score coefficients are used to calculate the component scores for a specific set of values of variables respectively characteristics. This capability will be used later on.

Generally, a high value of the component loadings or component score coefficients indicates a high influence of that characteristic on the respective component.

If the entry is positive, the characteristic and the component behave rectified to each other (the influence is proportional) and if the entry is negative, the characteristic and the component behave reverse to each other (the influence is disproportional). The results are interpreted in the next section, leveraging these relationships between component and characteristics.

\subsection{Interpretation}

The characteristics that cluster on the same components suggest that component 1 represents organisational complexity, component 2 product-related complexity, and component 3 inertia. By default, every characteristic contributes to every component.

Organisational complexity increases with the number of contractors/suppliers, employees, changes, disciplines, and the part count. It is decreased through a higher unit cost, which could indicate a more systematic development approach.

Product-related complexity increases with the number of disciplines, part count, unit cost, and source lines of code. With unit cost and source lines of code being the dominant characteristics. It decreases with the number of contractors/suppliers and the number of changes. The first could be explained through an outsourcing of complexity, if contractors/suppliers are solely responsible for a module of the product. The latter is unintuitive but could be explained through the avoidance of product complexity by the development team, as a high product complexity would lead to a high change effort per change.

Inertia increases with the number of disciplines, part count, unit cost, and cycle time. With cycle time being the most dominant characteristic. It decreases with the number of contractors/suppliers, employees, and source lines of code. The first two could be justified by the workload being spread over more organisations and/or employees, increasing the organisation's agility. The latter is harder to justify, it could be that the short development cycle and integration capabilities in the software domain create this effect. Using the component score coefficients, the organisational complexity, product-related complexity and inertia can be computed using Equation (1) for a set of values of the characteristics.

$$
\text { Component score }_{i}=\sum_{j=1}^{8} \text { Component score coefficient }_{i j} \times \frac{\left(\text { Characteristic }_{j}-\mu_{j}\right)}{\sigma_{j}}
$$


The component score coefficients (Component score coefficient ${ }_{i j}$ ) are depicted in the component score coefficient matrix in Figure 3. Figure 4 shows the categorization of characteristics and related value of the characteristic (Characteristic C. $_{j}$.

\begin{tabular}{|c|c|c|c|c|c|c|c|c|}
\hline Value for characteristic & 1 & 2 & 3 & 4 & 5 & 6 & 7 & 8 \\
\hline 1 Number of contractors/suppliers & 0 & 1 & $1-5$ & $5-20$ & $20-50$ & $50-100$ & $100-1.000$ & $>1.000$ \\
\hline 2 Number of employees & $1-10$ & $10-50$ & $50-100$ & $100-500$ & $500-1.000$ & $>1.000$ & - & - \\
\hline 3 Number of changes & 0 & 1 & $1-5$ & $5-10$ & $10-20$ & $20-100$ & $>100$ & - \\
\hline 4 Number of disciplines & 1 & $1-5$ & $5-10$ & $10-20$ & $20-30$ & $30-50$ & $>50$ & - \\
\hline 5 Part count & 0 & $1-10$ & $10-100$ & $100-1.000$ & $1.000-10.000$ & $10.000-100.000$ & $100.000-1.000 .000$ & $>1.000 .000$ \\
\hline 6 Unit cost in \$ & $1-100$ & $100-1.000$ & $1.000-10.000$ & $10.000-100.000$ & $100.000-1.000 .000$ & $1.000 .000-10.000 .000$ & $>10.000 .000$ & - \\
\hline 7 Source lines of code & 0 & $1-10$ & $10-100$ & $100-1.000$ & $1.000-10.000$ & $10.000-100.000$ & $100.000-1.000 .000$ & $>1.000 .000$ \\
\hline 8 Cycle time & $<1$ & $1-6$ & $6-18$ & $18-36$ & $36-60$ & $60-120$ & $>120$ & - \\
\hline
\end{tabular}

Figure 4. Categorization of characteristics and related value of the characteristic

The specific mean $\left(\mu_{j}\right)$ and the standard deviation $\left(\sigma_{j}\right)$ for each characteristic are shown in Figure 5.

\begin{tabular}{|c|lc|c|}
\hline $\mathbf{j}$ & Variable & $\boldsymbol{\mu}_{\boldsymbol{j}}$ & $\boldsymbol{\sigma}_{\boldsymbol{j}}$ \\
\hline $\mathbf{1}$ & Number of contractors/suppliers & 4,82 & 1,673 \\
\hline $\mathbf{2}$ & Number of employees & 3,70 & 1,584 \\
\hline $\mathbf{3}$ & Number of changes & 4,53 & 1,496 \\
\hline $\mathbf{4}$ & Number of disciplines & 4,09 & 1,504 \\
$\mathbf{5}$ & Part count & 5,14 & 1,633 \\
\hline $\mathbf{6}$ & Unit cost in \$ & 4,93 & 1,787 \\
\hline $\mathbf{7}$ & Source lines of code & 5,91 & 1,882 \\
\hline $\mathbf{8}$ & Cycle time & 4,43 & 1,448 \\
\hline
\end{tabular}

Figure 5. Standard deviation and specific mean for each characteristic

As all component scores are positively correlated with each other, meaning that the individual complexities increase with each other, we can use Equation (2) as a first approximation to estimate a total complexity score, simplifying further analyses.

$$
\text { Total complexity }=\sum_{i=1}^{3}{\text { Component } \text { score }_{i}}
$$

\subsection{Product-related and organisational complexity drive the adoption of MBSE}

As literature showed, there is an untested assertion in the MBSE community that complexity drives the adoption of MBSE. Analysis of the survey data supports this assertion.

\section{What feature or features do you believe have made the aerospace industry a leader in MBSE? (Select all that apply.)}

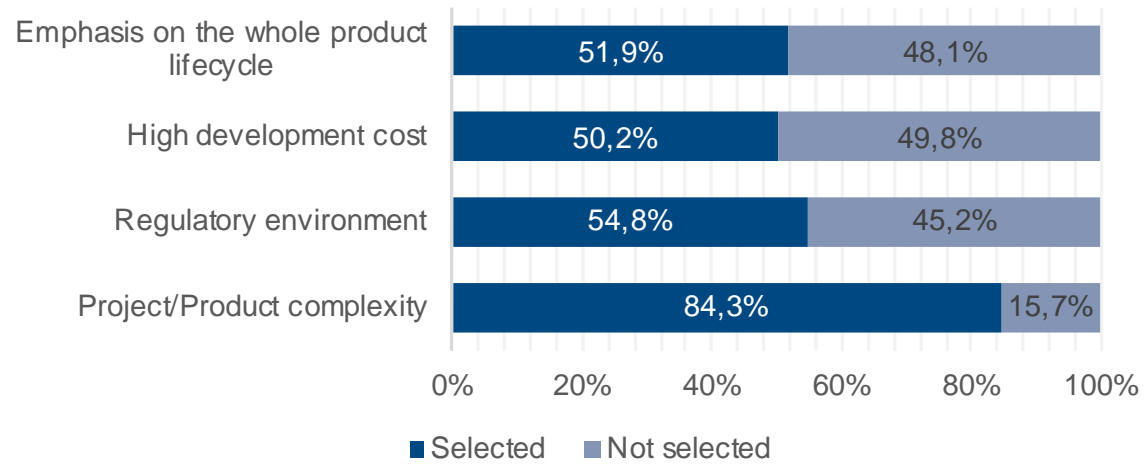

Figure 6. Feature or features that allegedly have made the aerospace industry a leader in MBSE $(\mathrm{N}=715)$ 
The participants of the course were asked in a multiple-choice question what feature or features they believe have made the aerospace industry a leader in MBSE. Provided answer possibilities were project/product complexity, regulatory environment, high development cost, and emphasis on the whole product lifecycle. The results can be seen in Figure 7.

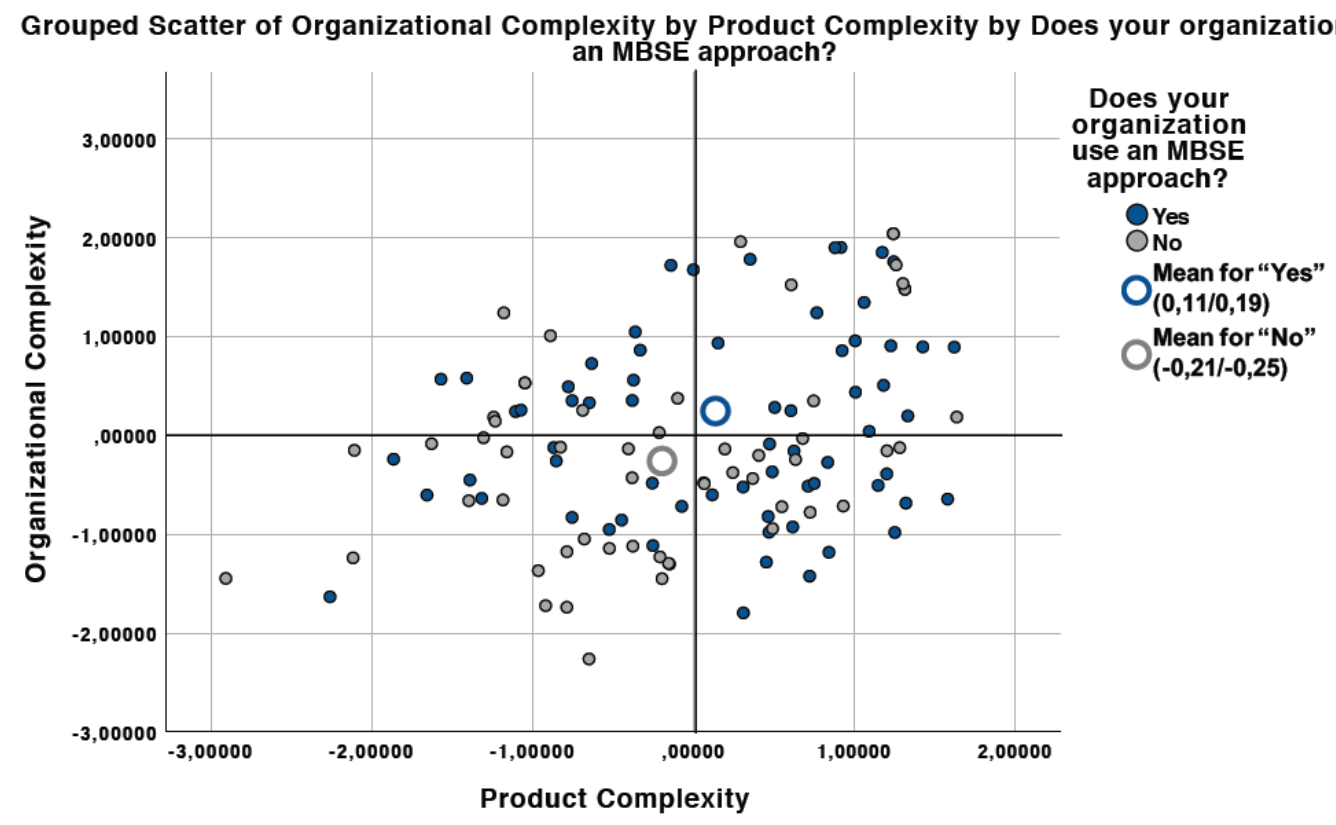

\section{Figure 7. Effect of organisational and product-related complexity on the number of MBSE adopters}

$85 \%$ of the 715 participants believe that project/product complexity have made the aerospace industry a leader in MBSE. With the other answer possibilities ranging around an approval to disapproval rate of 1 , this seems to represent a significant consensus. Indicating, that the participants see complexity as the main driver for MBSE adoption, as well.

Using the defined complexities and total complexity, the general assertion that complexity drives the adoption of MBSE is strongly supported. A t-test of the total complexity and the usage of MBSE showed a significant difference in means. The difference being that organisations using MBSE experience significantly greater total complexity than organisations not using MBSE. Remembering the value proposition of MBSE (to support multiple activities in the system lifecycle), it can be assumed that organisations experiencing greater total complexity need more support in these activities and therefore derive more benefit indicated through the proportionality of adoption rate and complexity from using MBSE. Further investigating each component revealed, that while t-tests showed a significant difference in means for product-related complexity and an almost significant (on a $\mathrm{p}<0,05$ level) difference in means for organisational complexity (both were proportional to the number of adopters), inertia had no significant effect on the number of adopters. Figure 6 visualizes the effect of organisational and product-related complexity on the number of MBSE adopters. The result supports the general assertion that complexity drives the adoption of MBSE.

\subsection{Evaluation of achievable potential though adopting MBSE}

System engineers and managers initially evaluating the achievable potential through an MBSE adoption can use formula 1 and 2 to calculate the complexity scores and total complexity for their respective organisational settings. This will allow them to classify their organisation regarding the scores of the white goods, automotive, aerospace and defence industry described and discussed below. This can lead to a better-informed decision about whether MBSE should be further investigated as a candidate for a digital engineering methodology. Figure 8 shows the industry means for productrelated, organisational and total complexity in the survey sample. 

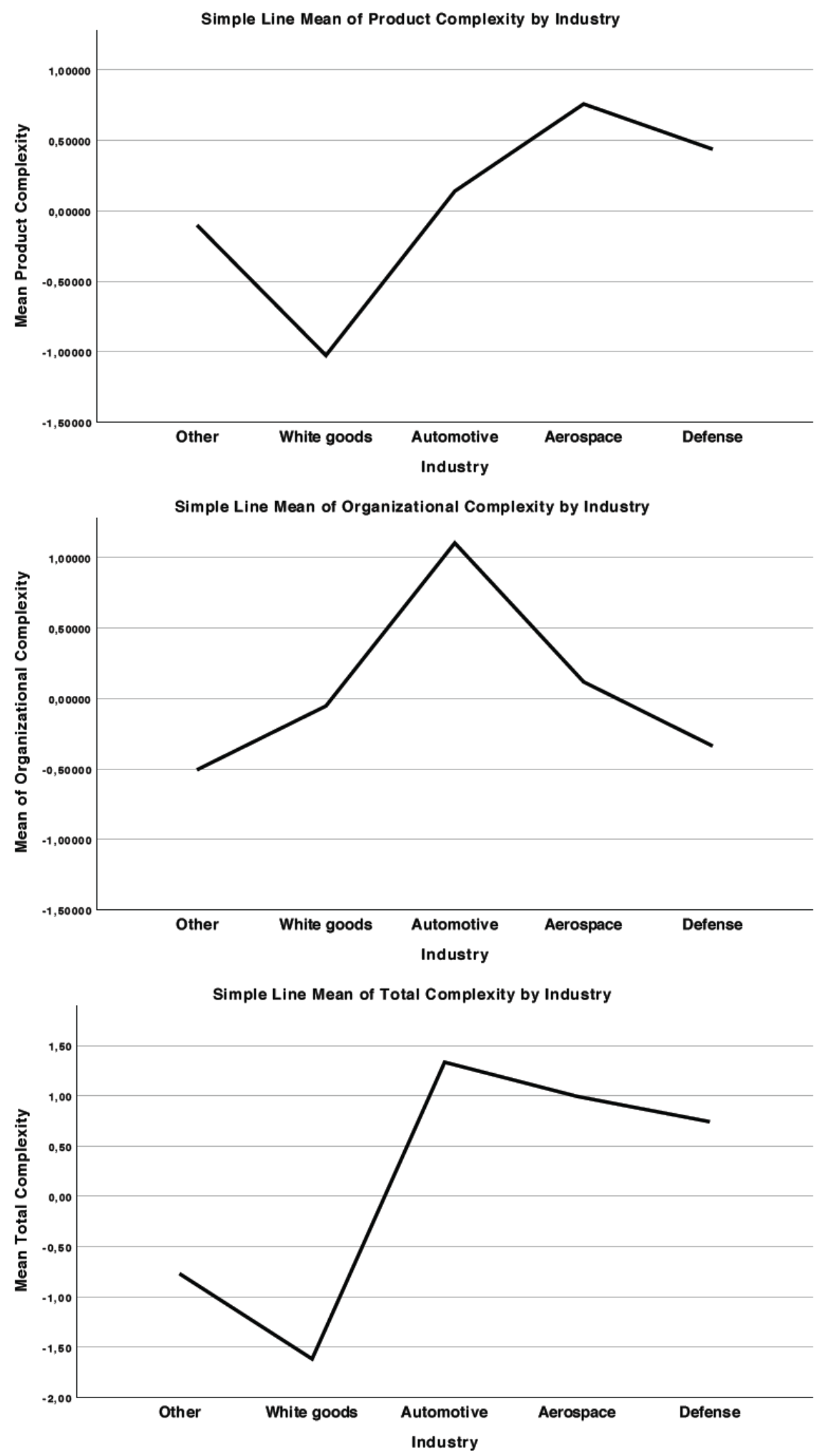

Figure 8. Industry means for product-related, organisational and total complexity

Product complexity increases from the white goods, automotive, defence to the aerospace industry. Significantly high organisational complexity prevails in the automotive industry. In the white goods and aerospace industry, organisational complexity is not decisive. Defence and other industries experience low organisational complexity compared to everyone else. The total complexity is high in the automotive, aerospace and defence industry, while it is low in the white goods industry. 
Earlier it was shown that complexity drives the adoption of MBSE. Using Figure 8 managers can compare their organisation's complexity scores with other industries. If their respective organisation's characteristics classify them well above average $(>\sim 0.3)$ for at least one of the specific complexities (product-related or organisational complexity), they should strongly consider the use of MBSE. In their organisation's complex setting MBSE is an appropriate methodology to cope with the negative effects of increased complexity.

\section{Discussion, conclusion and outlook}

The insights from the survey imply a high-level of cogency. This stems from the use of results from significant statistical analysis carried out on a big dataset obtained from a heterogenous and qualified sample. Nonetheless, the validity of all insights from the survey is limited to the survey sample. Extrapolating them falls under the assumption that the survey sample sufficiently represents the affected population.

Literature studies revealed a general assertion in the model-based systems engineering community that complexity drives the adoption of MBSE. Through deriving components driving the adoption of MBSE from product and development task characteristics using a PCA, the complexity relationship was supported. Furthermore, the component scores allow practitioners to categorize their organisations setting in terms of complexity. This can help managers to initially evaluate the potential benefits of adopting MBSE.

During the study and in expert discussions it became clear that there is no unified understanding of MBSE. While this unified understanding should be a target for the active MBSE community, the paper at hand provides decision makers with the opportunity to assess an MBSE adoption without having a full understanding of the methodology.

Regarding current trends and directions of MBSE, the results presented in this paper suggest that further methodological developments should focus on the management of product-related and organisational complexity factors, as most of the adopters experience these two.

In the future, the initial evaluation approach should be extended to cover a cost-value or cost-benefit estimation and the drivers of the MBSE adoption will be examined on a more detailed level.

\section{References}

Field, A. (2009), Discovering Statistics Using SPSS, Thousand Oaks, CA, sage, 9781847879073.

Friedenthal, S., Moore, A. and Steiner, R. (2015), A practical guide to SysML: the systems modeling language, Burlington, MA, Morgan Kaufmann. 0128008008

Huldt, T. and Stenius, I. (2019) "State-of-practice survey of model-based systems engineering", Systems Engineering, Vol. 22, pp. 134-145. https://doi.org/10.1002/sys.21466

Hutcheson, G.D. and Sofroniou, N. (1999), The multivariate social scientist: Introductory statistics using generalized linear models, Thousand Oaks, CA, Sage. 0761952012

INCOSE (2007), Systems engineering vision 2020, San Diego, CA, INCOSE. Available at: http://www.ccose.org/media/upload/SEVision2020_20071003_v2_03.pdf

INCOSE (2014), A world in motion: systems engineering vision 2025, San Diego, CA, INCOSE. Available at: https://www.incose.org/docs/default-source/aboutse/se-vision-2025.pdf

Madni, A.M. and Purohit, S. (2019), "Economic Analysis of Model-Based Systems Engineering”, Systems, Vol. 7, pp. 1-18. https://doi.org/10.3390/systems7010012

Madni, A.M. and Sievers, M. (2018), "Model-based systems engineering: Motivation, current status, and research opportunities", Systems Engineering, Vol. 21, pp. 172-190. https://doi.org/10.1002/sys.21438

Reichwein, A. and Paredis, C. (2011), "Overview of architecture frameworks and modeling languages for model-based systems engineering", Proceedings of the ASME 2011 International Design Engineering Technical Conferences \& Computers and Information in Engineering Conference, 2011, New York, NY, pp. 1-9.

Scheeren, I. and Pereira, C.E. (2014), "Combining Model-Based Systems Engineering, Simulation and Domain Engineering in the Development of Industrial Automation Systems: Industrial Case Study", IEEE 17th International Symposium on Object/Component/Service-Oriented Real-Time Distributed Computing, IEEE, 2014. pp. 40-47. https://doi.org/10.1109/ISORC.2014.64

Sheard, S. et al. (2015), "A complexity primer for systems engineers", INCOSE Complex Systems Working Group White Paper, Vol. 1, pp. 1-10. 
